\title{
The Need for Visits to Social and Vocational Programs for the Mentally Ill as Part of General Psychiatry Residency Training
}

\author{
Ahsan Y. Khan, M.D. ${ }^{1}$, Rachna Kalia, M.D. ${ }^{2}$, \\ Elizabeth Ablah, Ph.D., M.P.H. ${ }^{3}$, Andrew Foote, M.D. ${ }^{1}$ \\ ${ }^{1}$ University of Oklahoma Health Sciences Center \\ Department of Psychiatry \& Behavioral Sciences, Oklahoma City, OK \\ ${ }^{2}$ South Central Mental Health, Andover, KS \\ ${ }^{3}$ University of Kansas School of Medicine-Wichita \\ Department of Preventive Medicine and Public Health, Wichita KS
}

\begin{abstract}
Background. Comprehensive treatment planning for psychiatric illnesses should be based on a biopsychosocial model of treatment to address the acuity and chronicity of these disorders. To achieve this goal, knowledge about pharmacological, psychological, and social aspects of the treatment plan should be presented as an integral part of general psychiatry residency training. This survey study was conducted to examine how many programs provide training where residents have scheduled visits to social and vocational mental health service organizations in the community and to identify potential obstacles to including this rotation in general psychiatry residency training.

Methods. A voluntary, anonymous survey was sent via SurveyMonkey ${ }_{\circledast}$ to the program directors of all general psychiatry residency programs in the United States. The survey consisted of five questions designed to assess if their programs had a rotation where residents visit social and vocational programs in the community designed for mentally ill patients to provide knowledge of the community mental health resources to their residents.

Results. Of the 168 survey invitations issued, 73 (44\%) responded. Fifty-six responders acknowledged that their residents were required to visit a community mental health organization, but their programs did not offer visits to community social and vocational programs. Seventeen program directors reported that their program did not provide this experience to their residents and indicated a desire to include such a rotation.

Conclusions. Community mental health service organization visits should enhance knowledge of psychiatry residents about community mental health resources and indirectly promote better patient care. Information obtained from this survey should create discussion to work toward better psychiatric resident training.

KS J Med 2014; 7(2):39-45.
\end{abstract}

\section{Introduction}

The management of patients with mental illness is multifaceted and involves the understanding of biopsychosocial factors contributing to the acuity and chronicity of the illness. Although many talk about the biopsychosocial model, few practice it regularly. ${ }^{1}$ In an era of managed care, when inpatient hospitalization is getting shorter, and recovery-oriented mental health care has become predominant, it makes sense to acquire knowledge about the tools used to provide social interventions. Therefore, the training of a psychiatrist must provide knowledge about the biological and psychological treatment options and social interventions which are an integral part of the biopsychosocial treatment model to treat psychiatric conditions. 
The Accreditation Council for Graduate Medical Education (ACGME) is the major organization regulating residency training programs throughout the United States. The concept of the biopsychosocial model is central to the ACGME Program Requirements for Graduate Medical Education in Psychiatry. ${ }^{2}$ Indeed, programs must annually evaluate residents in the Postgraduate (PG)2, PG-3, and PG-4 years and "conduct and examination across biological, psychological, and social spheres...”. ACGME requirements for community psychiatry training within the United States states that "this experience must expose residents to persistently and chronically-ill patients in the public sector (e.g., community mental health sectors, public hospitals and agencies, and other community-based settings). The program should provide residents the opportunity to consult with, learn about, and use community resources and services in planning patient care, as well as to consult and work collaboratively with case managers, crises teams, and other mental health professionals."

Despite these recommendations from ACGME, most psychiatric training programs emphasize psychopharmacology, laboratory values, and imaging studies as part of the "biological" approach to treating mental illness. Psychiatrists may feel that they are marginalized as persons who only prescribe medications, therefore, are following a reductionist approach in the treatment of their patients. ${ }^{3}$

In 1986-1987, the American Association of Community Psychiatrists (AACP) conducted a survey of psychiatry residency training programs, which gathered data regarding current practices and trends in training residents in "social, community, or public" aspects of psychiatry. ${ }^{4}$ Using a checklist format, they asked residency programs about clinical settings used for training, treatment modalities, patient populations, and topics covered in didactic coursework. The survey focused primarily on the existence of an "identifiable program of social and community psychiatry" as part of the clinical rotation. Survey results indicated that $92 \%$ of the respondent programs had some form of community mental health center available for training.

A more intensive model called the "public psychiatry module" was developed in 1991 at the University of Miami Medical School. ${ }^{5}$ They pointed out that "a rotation in a community mental health center (CMHC) may not adequately educate residents about the interlocking network of services available to patients and the large array of elements necessary for an effective continuum of care." Sobhan et al. ${ }^{6}$ concluded that "residents would need more than a rotation of 6 months' duration in a community mental health center to learn about the interlocking network of services available to patients and the large array of elements necessary for an effective continuum of care."

Emphasizing the social aspect of treatment is long overdue. Each ACGMEaccredited psychiatry residency training program has developed its own unique systems of providing their residents with this experience. Some training programs may approach it by imparting theoretical knowledge in the form of didactics and involving the social worker in a typical inpatient-based treatment planning alongside residents. However, it is doubtful if this approach can match real-life exposure to diverse service delivery settings available in the community that truly reflect the complex systems involved in the treatment of mentally ill people.

At the University of Kansas School of Medicine-Wichita, monthly scheduled educational tours to different community mental health service organizations focus on 
the social and vocational programs in the community specifically designed for mentally ill patients. These visits are scheduled in the third year as it is the principal outpatient year of training and residents are responsible to provide a comprehensive treatment plan for their patients by following the biopsychosocial treatment approach. These mental health service organizations pertain to giving mental health patients a richly enmeshed network of "wrap around" services so that their social milieu is shaped to have the best outcome.

These monthly educational tours range in duration from 1 to 2 hours under faculty supervision. During scheduled tours, the facility staff usually presented information about their facility and type of services offered. Residents asked questions about the facility, the type of services provided, funding sources for the facility, requirement for medical insurance coverage for their members, their plan in case of emergency, the type of staff available on-site, any fee involved, any job opportunities/placement help available for their members, how to refer patients for their facility, and whether any rehabilitation services are provided, including short stay. The community sites varied in terms of the diversity of the services offered as well as the nature of the clients they serve. Examples of sites included: vocational rehabilitation places for people with physical and mental disabilities, social clubs for people with severe and persistent mental illness, mental health clinics located in jails, local mental health associations, inpatient drug and alcohol rehabilitation service providers, and nonprofit organizations for children with special needs. Tours were in addition to the actual clinical rotations that residents complete at a local community mental health center (CMHC), one day/week throughout their third year of training.
The present study examined the need for visits to social and vocational programs as part of general psychiatry residency training. Programs were asked to share their reasons for providing or not providing such educational tours. This particular array of the "community" aspect of psychiatry training was studied for several reasons. To our knowledge, such a study has not been conducted previously. We wanted to assess other programs' viewpoints regarding our self-constructed approach to target the lacuna of training in community services. Finally, we sought to assess the outcomes from programs including such tours.

\section{Methods}

Prior approval was obtained from the Institutional Review Board (IRB) at the University of Kansas School of MedicineWichita (KUSM-W).

Participants. All general psychiatry residency program directors who were listed on the FREIDA database on the American Medical Association website were emailed directly from SurveyMonkey ${ }_{\circledast}$ to complete an assessment $(\mathrm{n}=175)$. Each e-mail contained an embedded link to the anonymous survey.

Survey. A brief, five-question survey was designed to assess residents' exposure to community mental health services via visits or educational tours of community mental health service organizations in their area (Table 1). Program directors were asked to indicate if their residency program had a requirement for residents to visit or participate in educational tours. The programs which reported having this requirement were asked to discuss the outcomes of including visits or tours in their programs. The other residency directors were asked about their reasons for not having such a requirement. 
Table 1. Survey questions.

1. Does your psychiatric residency program include a component that requires residents to visit or go for educational tours to community mental health service organizations or mental healthoriented social/vocational training programs?

2. What positive outcomes have you seen from exposing your residents to area mental health service organizations or social/vocational training programs?

3. Would you like to include such visits or education tours in your program?

4. Why not?

5. What has kept you from including educational tours to area mental health service organizations?

Data analysis. Survey data were downloaded from SurveyMonkey ${ }_{\circledast}$ into Microsoft Excel before being imported into Statistical Packages for the Social Sciences (SPSS) Version 15 (SPSS Inc., Chicago, IL) for analysis. Univariate analyses were conducted on the quantitative data, with frequencies tabulated to describe responses. Pattern coding was utilized to characterize qualitative responses. This analysis option was utilized due to its ability to pull large amounts of content together into more meaningful and parsimonious units of analysis.

Qualitative responses to the items were reviewed to identify the most prevalent pattern or themes among responses, and then coded and sorted. Research team members independently viewed the qualitative and quantitative results. These separate results were compared in conference among researchers so that final recommendations for coding could be made.

\section{Results}

Of the 175 surveys distributed, seven were returned due to invalid email addresses, resulting in a final pool of 168 potential respondents. Of these, 44\% ( $\mathrm{n}=$ 73) responded to the survey. Fifty-six respondents (77\%) reported that their residency program included a component that requires residents to visit or participate in educational tours of community mental health service organizations or mental health oriented social/vocational training programs. Seventeen respondents (23\%) reported their psychiatric residency programs did not include such a component requiring residents to visit or go on educational tours. Eleven programs (65\%) without such requirements indicated a desire to include such a requirement in the future.

Of the 56 respondents that reported that their residency program requires visits or offers educational tours, 48 (86\%) respondents offered comments about the positive outcomes associated with these visits. Responses were broadly categorized into three thematic categories: a) a better appreciation/awareness for community mental health service organizations in terms of their nature, logistics, operation and services $(n=24)$; b) an increased interest in community mental health organizations as prospective employers for residents ( $\mathrm{n}=$ 15); and c) better ability to work with their patients with improved knowledge about area mental health service organizations ( $\mathrm{n}=$ 5). The remaining responses were categorized as miscellaneous $(\mathrm{n}=4)$ including responses where program directors have confused these tours with their regular community mental health clinic rotations.

Six respondents reported that they would not like to include such visits or educational tours in their program. Five of these respondents reported their reasons for not wanting educational tours. The reasons included that residents already are assigned to community mental health centers $(n=3)$, few patients take advantage of community resources $(n=1)$, and a perception of the 
local community mental health center being a "disaster" $(\mathrm{n}=1)$.

The 11 programs who did not require residents to visit community mental health service organizations, but would like to do so, reported the barriers that have kept them from adding such a rotation. All reported time constraints as their main reason. The common constraints were organizing and scheduling the tours $(\mathrm{n}=5)$, lack of planning and availability of faculty $(\mathrm{n}=3)$, and lack of program organization and community sponsorship $(\mathrm{n}=2)$.

\section{Discussion}

The survey specifically focused on scheduled visits/tours to community mental health service organizations by residents under faculty supervision to acquire knowledge about community resources. The overall response rate of our survey was comparable with the return rate of a similar survey. ${ }^{4}$ In fact, the previous survey identified some sites, such as homeless shelters, intellectual and developmental disabilities programs, jails, and halfway houses, as unavailable to residents, even as electives. In contrast, these sites are prime examples of the psychosocial visit sites asked of our survey respondents.

Fifty-six respondents reported that their residency programs required residents to visit or participate in educational tours of community mental health service organizations. However, based on respondent survey comments, a majority had a CMHC rotation where residents see patients rather than educational tours for couple of hours every month to understand their operation, process of referral, and type of patients who can be referred. Since the majority (77\%) of respondent programs required their residents to go on such educational tours, it may be possible that some of these programs misunderstood the term "area mental health service organizations" as being equivalent to a $\mathrm{CMHC}$ or confused their regular rotations at a CMHC to monthly scheduled tours/visits to different mental health service organizations. If that was the case, the $77 \%$ could be an inflated estimate. Thus, the present findings should be considered preliminary and warrant further delineation of the exact meaning of such terms used in any future surveys.

Failing to identify respondents geographically was another limitation of this survey. The survey was intentionally anonymous. Anonymity was preserved to avoid influencing public opinion for any particular program. Also, anonymous surveys were more likely to be completed by program directors.

Another major limitation of our survey was the lack of an operational definition of terms such as "community mental health service organizations" or "mental healthoriented social/vocational training programs". A thorough literature search failed to yield such a clear definition. Widespread confusion regarding what constitutes a community mental health center has been documented on more than one occasion. ${ }^{7-10}$ The lack of a sharply defined definition and theoretical matrix was discussed at the 153rd Annual Meeting of the American Psychiatric Association, when Kenneth Thompson, M.D., convened a session to address the future of community psychiatry in the United States. ${ }^{11}$ To address this issue, the AACP has embarked on a Community Psychiatry certification initiative. $^{12,13}$

In addition to the lack of a clear concept of "community mental health", there is a well-documented deficiency of a single, well-defined, core curriculum for psychiatry training programs, despite numerous attempts to develop one. As the development of a single universal curriculum may not be practical in light of 
local availability of resources, residency programs could benefit from sharing other ways of implementing training. Past research has suggested making curricula available on residency program websites. ${ }^{6}$

\section{Conclusion}

This survey was intended to create a "snapshot" of US psychiatry residency programs' views about including educational tours to community mental health service organizations in their residency curricula. Far-reaching conclusions cannot be drawn from the results of this pilot survey. However, the need to explore this aspect of psychiatry training further is evident. One necessary improvement for future surveys includes specific definitions of terms such as "community mental health service

\section{References}

${ }^{1}$ Liberman RP, Glick ID. Drug and psychosocial curricula for psychiatry residents for treatment of schizophrenia: Part I. Psychiatric Serv 2004; 55(11):1217-1219. PMID: 15534008.

2 Accreditation Council for Graduate Medical Education. ACGME Program Requirements for Graduate Medical Education in Psychiatry. Available at: http://www.acgme.org/acgmeweb/Portals/ 0/PFAssets/ProgramRequirements/400_ps ychiatry_07012007_u04122008.pdf.

${ }^{3}$ Rogers JA, Vergare MJ, Baron RC, Salzer MS. Barriers to recovery and recommendations for change: The Pennsylvania Consensus Conference on psychiatry's role. Psychiatr Serv 2007; 58(8):11191123. PMID: 17664525.

${ }^{4}$ Goldman CR, Brown DB, Thompson KS. Community psychiatry training for general psychiatry residents: Results of a national survey. Community Ment Health J 1993; 29(1):67-76, 77-83. PMID: 8448981.

${ }^{5}$ Brauzer B, Lefley HP, Steinbook R. A organizations", "mental health-oriented social/vocational training programs", and "educational tours". It also may be worthwhile to obtain more detailed information about the amount of time allotted for tours/visits, their locations, and the residents' level of training. Such questions will help to identify nationwide themes, which can be used as building blocks for community psychiatry training.

Community mental health service organizations visits should enhance knowledge of psychiatry residents and indirectly promote better patient care. Information such as obtained from this survey should create discussion to work toward better psychiatric resident training. In our program, consistently positive feedback from the residents has kept educational tours available for several years.

module for training residents in public mental health systems and community resources. Psychiatr Serv 1996; 47(2):192194. PMID: 8825259.

${ }^{6}$ Sobhan T, Husain Z, Rahman A, Feldman JM, Dahl DC. Residency training in public psychiatry: A review of literature. J Ky Med Assoc 2004; 102(7):307-314. PMID: 15291133.

${ }^{7}$ Mollica RF. From asylum to community. The threatened disintegration of public psychiatry. N Engl J Med 1983;308(7): 367-373. PMID: 6823241.

${ }^{8}$ Panzetta AF. Whatever happened to community mental health: Portents for corporate medicine. Hosp Community Psychiatry 1985; 36(11):1174-1179. PMID: 4065841.

${ }^{9}$ Group for the Advancement of Psychiatry. Community Psychiatry: A Reappraisal. New York, NY: Mental Health Materials Center, Group for the Advancement of Psychiatry, 1983. ISBN-10: 0910958181.

${ }^{10}$ Talbott JA, Bray JD, Flaherty L, 
Robinowitz C, Taintor Z. State-university collaboration in psychiatry: The Pew Memorial Trust Program. Community Mental Health J 1991; 27(6):425-439. PMID: 1773600.

${ }^{11}$ Cohen CI, Feiner JS, Huffine C, Moffic HS, Thompson KS. The future of community psychiatry. Community Ment Health J 2003; 39(5):459-471. PMID: 14635987.

${ }^{12}$ McQuistion H. President's Column. May 2009. Community Psychiatrist 2009; 23(1):1-2. Available at: http://www. communitypsychiatry.org/news/newsletter /AACPNewsV23N1.pdf.

${ }^{13}$ Kotwicki RJ, Compton MT. Key features of a unique community psychiatry fellowship: The Emory University fellowship in community psychiatry/public health. Community Ment Health J 2010; 46(4):403-408. PMID: 19662529.

Keywords: community psychiatry, internship and residency, mentally ill persons, community-institutional relations 